\title{
Cuidados paliativos no significa final de los tiempos
}

Luz Marina Cano Molano ${ }^{1}$

1 MD, Mg Educación, Ph.D. Doctorante en Pensamiento Complejo. Docente investigadora de la Fundación Universitaria Juan N. Corpas. Este artículo está basado en el trabajo investigativo desarrollado en el grupo de investigación Complexus.
Correspondencia: Dra. Luz Marina Cano Molano,luz.cano@juanncorpas.edu.co

Como citar: Cano LM. Cuidados paliativos no significa final de los tiempos. Cuarzo 2015; 21 (2): 61-67.

Recibido: 10 de septiembre de 2015 Aceptado: 25 de noviembre de 2015 Publicado: 15 de diciembre de 2015

Licencia creative commons (c) (i) $\Theta$

\section{Resumen}

Estudios recientes han develado que los equipos de atención primaria en salud no se encuentran capacitados para enfrentar el paciente moribundo, su familia y mucho menos preparados para el manejo adecuado de la verdad y toma de decisiones al final de la vida. Varios son los conflictos a los que se ven enfrentado el equipo sanitario ante la enfermedad terminal, al contrario de los problemas que se enfrentan en cualquier ciclo vital humano, el final de la vida requiere de un grupo transdisciplinar que aborde las diferentes fases del paciente, la familia y el mismo personal de salud1,19. La inseguridad del equipo por falta de conocimiento en Cuidados Paliativos (CP) se traslada a la prolongación del sufrimiento del paciente y su micromundo. Las estadísticas sobre investigaciones en CP, han demostrado que el desarrollo de los cuidados paliativos y los sistemas nacionales de salud están relacionados con el ingreso per cápita, en otras palabras, para mayor bienestar en mayor el gasto económico, especialmente al final de la vida4. En este artículo se reflexiona sobre esta situación, teniendo en cuenta que el 32\% de los países aún no forman a los profesionales en salud médico, enfermera, psicólogo, nutricionista, trabajo social en cuidados paliativos ni el pregrado ni posgrado, se deja esta práctica a voluntarios o cuidadores de hogares de la tercera edad o cuidado especial y aunque el personal sanitario está formado para cuidar, esta función se ha olvidado y de allí parten las innumerables quejas por abandono terapéutico.

KEY WORDS: cuidados paliativos, atención primaria, formación.

Abstract
Palliative care does not mean the end of time
Recent studies have revealed that teams of primary health care
are not trained to deal with the dying patient, family and much less
prepared for the proper handling of truth and decision making at the
end of life. There are several conflicts that are facing the healthcare
team before the terminal illness, unlike problems faced in any human
life cycle, the end of life requires an interdisciplinary group that ad-
dresses the different stages of the patient, family and staff salud1,19
same. Team insecurity due to lack of knowledge in Palliative Care


(CP) moves to the continued suffering of the patient and microworld. Statistics on research in CP, have shown that the development of palliative care and national health systems are related to per capita income, in other words, greater welfare greater economic spending, especially at the end of the life.4. This article reflects on this situation, considering that $32 \%$ of countries have not yet become professionals in medical health, nurse, psychologist, nutritionist, social work in palliative care or undergraduate or graduate, this practice is allowed volunteers or caregivers of elderly homes or special care and even health staff are trained to care for, this feature has been forgotten and there leave the innumerable complaints therapeutic abandonment.

KEYWORDS: academic writing skills and communication skills, organizational strategies.

\section{Introduction}

Actualmente, las poblaciones están aumentando y proporcionalmente la tercera edad, así como el promedio de vida. En las siguientes décadas aumentarán los pacientes en fase terminal por enfermedad oncológica, patología infecciosa o no transmisible gracias a los avances tecnológicos y tratamientos fútiles. Se espera que para el 2030, la mortalidad del cáncer pase de 12 millones a 20 millones y el $80 \%$ de los pacientes pertenecen a estratos socioeconómicos bajos con acceso y recursos de salud limitados. Estas cifras han resaltado la necesidad de entrenamiento y educación de todo el personal sanitario y cuidadores de hogar para afrontar asertivamente las fases finales de la enfermedad; así como la creación de políticas en salud exclusivas del cuidado paliativo; así como la investigación en este campo ya que la literatura y evidencia son limitadas $(1,3)$.

Ante el incremento de pacientes que lo requieran en nuestras latitudes, es necesario investigar localmente e insertar este tema en el currículo como cátedra, clínicas o grupos de trabajo en pre y pos grado, y particularmente de la cuestión: cuál es la consecuencia, para el paciente en términos de prolongación del sufrimiento, para la familia, para las IPS, para el sistema de salud, que los médicos o enfermeras colombianos no sepan de CP.

La Real Academia de la Lengua Española define la palabra paliar viene del latín tardío Palliare y el del latín Pallium que significa manto. Su traducción es mitigar la violencia de algunas enfermedades o atenuar una pena. La Organización Mundial de la Salud (OMS) define los Cuidados Paliativos (CP) como el acercamiento que mejora la calidad de vida de los pacientes y sus familias que enfrentan problemas asociados a enfermedades terminales mediante la prevención y alivio del sufrimiento, detectando y tratando impecablemente el dolor y otros síntomas asociados, así como problemas psicosociales y espirituales. Afirma la vida y considera la muerte como un proceso natural; no acelera ni pospone la muerte; integra los aspectos psicosociales y espirituales del paciente, su familia y cuidadores; ofrece soporte para que los pacientes vivan la más activamente posible hasta la muerte; ofrece soporte a la familia para afrontar la enfermedad y el duelo1.

En ese sentido se estima que cada año más de $20 \mathrm{mi}-$ llones de personas requieren cuidados paliativos (CP) al final de la vida: $67 \%$ por encima de los 60 años, $6 \%$ población infantil, 38\% por enfermedades cardiovasculares, $34 \%$ por cáncer, $10.3 \%$ por condiciones respiratorias y $5.7 \%$ por HIV/ SIDA. La muerte si bien es el final de la vida, se ha convertido en un tabú entre los profesionales de la salud y en algunos casos se considera el fracaso terapéutico, sin reconocer su valor en los servicios de urgencias, unidades intermedias y hogares de cuidado especial.

Infortunadamente, estudios recientes han develado que los equipos de atención primaria en salud no se encuentran capacitados para enfrentar el paciente moribundo, su familia y mucho menos preparados para el manejo adecuado de la verdad y toma de decisiones al final de la vida. Varios son los conflictos a los que se ven enfrentado el equipo sanitario ante la enfermedad terminal, al contrario de los problemas que se enfrentan en cualquier ciclo vital humano, el final de la vida requiere de un grupo transdisciplinar que aborde las diferentes fases del paciente, la familia y el mismo personal de salud (1,19). La inseguridad del equipo por falta de conocimiento en CP se traslada a la prolongación del sufrimiento del paciente y su micromundo. Las estadísticas sobre investigaciones en CP, han demostrado que el desarrollo de los cuidados paliati- 
vos y los sistemas nacionales de salud están relacionados con el ingreso per cápita, en otras palabras, para mayor bienestar en mayor el gasto económico, especialmente al final de la vida (4). El Reino Unido, Australia y Nueva Zelanda tienen una mejor calidad de vida, gracias a las políticas e integración extensiva de los CP al sistema de salud. Existen otras condiciones igualmente importantes, como el desarrollo e implementación de políticas en salud específicas en CP, presupuesto para los servicios de salud, entrenamiento continuo y de alta calidad para los equipos sanitarios, subsidio a las familias del paciente terminal, disponibilidad de opioides y educación comunitaria y a ciudadores $(4,19)$.

Por otra parte, si bien es una política de la OMS la integración de los CP a los sistemas de salud, muchos países ni siquiera conocen el concepto de los cuidados paliativos. Esto habla de la existente ruptura entre la asistencia y la academia. El 32\% de los países aún no forman a los profesionales en salud médico, enfermera, psicólogo, nutricionista, trabajo social en cuidados paliativos ni el pregrado ni posgrado, se deja esta práctica a voluntarios o cuidadores de hogares de la tercera edad o cuidado especial y aunque el personal sanitario está formado para cuidar, esta función se ha olvidado y de allí parten las innumerables quejas por abandono terapéutico (4). Según lo anterior, aunado a las estadísticas de la OMS, se están abriendo los espacios de aprendizaje en los currículos para la integración de los mismos como eje estructural de la formación en salud (5). Estudios han demostrado que la calidad de vida de los pacientes mejora con personal entrenado y asimismo mejora la economía del sistema de salud gracias a una mejor administración de los recursos, razón por la cual un número importante de instituciones están haciendo un llamado a la comunidad educativa para la implementación de los CP en los planes de estudio no solo en la formación médica., sino a las disciplinas que son participantes activos en la salud y a los que aún no lo han hecho1 (19).

\section{Situación actual de la formación en el cuidado paliativo}

Desde hace dos décadas Australia, implementó como política educativa junto con la Estrategia Nacional de CP y se incluyó en el currículo de pregrado. En los Estados Unidos, no hay CP en los planes de estudio de forma estandarizada y no son reconocidos por el Departamento de Educación y Acreditación, pero estudios han demostrado que varias facultades los han integrado satisfactoriamente. En 1993, en Canadá, se desarrolló en plan de estudios y competencias específicas y mediante la investigación ha logrado la internacionalización del mismo. En 1980, se consolidó la Asociación Europea de Cuidados Paliativos (AECP) y desarrolló las guías para formación y entrenamiento en el contexto multidisciplinar. Las recomendaciones fueron optimizadas para la creación del Task Force en CP en el 2011 y en el 2013 divulgadas (5), con seis dominios fundamentales: concepción de los cuidados paliativos, control del dolor y otros síntomas, aspectos psicosociales y espirituales, aspectos bioéticos y normativos, comunicación asertiva y trabajo en equipo, dimensiones homogéneas para las disciplinas en salud.

En Latinoamérica fue en la década de los 80, Colombia siendo el primero con el Hospicio la Viga y la clínica del dolor con la Universidad de Antioquia. En 1982, Argentina con la Liga de lucha contra el Cáncer; 1985, Uruguay, con el Hospital Saunders de la Asociación Española primera de los Socorros Mutuos; 1988, República Dominicana con el programa domiciliario de Cuidados Paliativos; 1989, Perú y México, con unidades de dolor; 1990, Chile, con asistencia domiciliaria;1992, Panamá, con la Asociación Hospices Pro cuidados Paliativos,1995, Cuba, Paraguay, Brasil y Ecuador con programas y unidades de CP; 2002, El salvador con el programa hospitalario de CP-Hospital de la Divina Providencia; 2006, Honduras con el Centro de Cáncer Emma Romero de Callejas y finalmente, en el 2007, Nicaragua (4).

Desde el 2006 se ha logrado la inclusión de los cuidados paliativos a los sistemas generales de salud, actualmente, hay $1,6 \%$ servicio/unidad/ equipo por cada 1.000.000 de habitantes en Latinoamérica, de los cuales el $0.9 \%$ hace parte de los niveles de atención primaria. Dentro de este último se destacan los hospicios; el 0,3\% centros de atención domiciliaria y $1 \%$ a las entidades de 2 y 3 nivel que tiene integrado este servicio. En Colombia, el 57 de las Unidades de Cuidados paliativos son de 3 nivel; 17\% Hospicios, 13\% equipos multinivel, 9\% servicios de atención domiciliaria, 4\% Unidades de segundo nivel y $1 \%$ equipos voluntarios.

En cuanto a los servicios acreditados, (4) los siguientes países cuentas con la misma: Costa Rica, 49 médicos espacialitas y 28 magister. Venezuela, 4 médicos espacialitas y 40 con Fellow. Colombia, 43 especialistas formados exclusivamente en universidades colombianas. Brasil, no 
había datos del número de médicos en esta disciplina para el momento de divulgación del Atlas. Los anteriores países reciben título de especialistas. Los siguientes reciben Certificación o Diplomado. Argentina, el certificado es otorgado por el Consejo de profesionales Médicos de la Academia Nacional de Medicina y para el 2013 habías más de 100. Chile, Este certificado lo otorgó el Ministerio de Salud entre 2006 y 2009 a más de 70 médicos. Panamá, el certificado es reconocido como un curso clínico avanzado. En Uruguay y Cuba, reciben diplomados en cuidados paliativos en el adulto mayor y control contra el cáncer respectivamente (4).

Ahora bien, existen tres países: Colombia, Uruguay y Cuba que cuentan con formación en pregrado en cuidados paliativos y ofrecen esta asignatura con horas u optativas. En cuba, en las 22 facultades de Medicina y 12 de enfermería, imparten en mismo plan de estudios para esta disciplina. Esta política también incluye los programas de psicología. En Uruguay, hay de facultades de Medicina acreditadas. Una incluye los CP de forma obligatoria en el plan de estudios y la segunda, 3 horas en la cátedra de psicología médica.

\section{Políticas sobre el cuidado paliativo}

En Europa, en el año 2000, el Convenio Económico, Social y Cultural de Derechos, pronunció como derechos fundamentales el cuidado de la salud en todos los individuos, acceso adecuado a fármacos y desarrollo de políticas de salud pública. Adicionalmente, en el 2006, el Papa Benedicto XVI consideró la preservación de la dignidad humana y defendió el cuidado del enfermo como derecho humano. En el 2011, la Asociación Europea de CP, publicó el "Reto Lisboa" que hace un llamado a las entidades gubernamentales a desarrollar políticas en salud para garantizar la satisfacción de las necesidades de los pacientes en fase terminal, eliminar las restricciones de opioides y otros fármacos esenciales para el confort, proveer entrenamiento adecuado a los actores de los servicios sanitarios de forma interdisciplinar y diseñar programas en salud autosostenibles en relación a la población y su comorbilidad (6). En el 2014, la Asociación Mundial de Cuidados Paliativos declaró el CP como un derecho humano. De forma particular en Latinoamérica, hay 3 Políticas en Salud establecidas como Leyes: Lay de Garantías de salud y Programa de alivio de dolor en Chile; Colombia, Ley 1722 del 2014 y en México la Ley de Materia en Cuidados Paliativos, Artículo 166 Bis (4).
En el año 2000, Ciudad de la Esperanza y al Asociación de Escuelas de Enfermería unieron esfuerzos para la creación del consorcio para la educación al final de la vida con el fin de formar profesionales de la salud para abordar las necesidades únicas del proceso de la muerte en sí. La meta era fortalecer el entrenamiento en el pregrado y posgrado con el programa entrenar a entrenadores. Una vez completado el curso, llevarían a sus comunidades su conocimiento para entrenar otros profesionales desarrollando el currículo con las siglas ELNEC (End of life nursing Education) (7).

Para el 2014, se estimó que había 7,600 individuos entrenados bajo este esquema curricular en 39 Estados Americanos. En otras palabras, el currículo de enfermería en cuidados paliativos tiene más de dos décadas y la sociedad internacional de enfermería divulgó los mínimos estándares académicos para el pregrado con dominios fundamentales que incluyen perspectivas globales de la muerte, control de síntomas, comunicación terapéutica, cuidado en las últimas horas de vida, pérdida, sufrimiento, duelo anticipado y bioética al final de la vida (8). Por su parte, en el 2004, La Asociación Latinoamericana de Cuidados Paliativos (ALCP) divulgó el currículo para la carrera profesional de enfermería como guía en la preparación académica de las enfermeras de Latinoamérica. Fue desarrollado para satisfacer la necesidad en enfermería de aprendizaje de Cuidado Paliativo y describe la capacitación necesaria para proveer asistencia de alta calidad. El currículo incluye diez módulos con temas relacionados con el control de síntomas, cuidados al final de la vida, aspectos psico-sociales y espirituales, ética, duelo, gestión e investigación. Además, contiene una sección con bibliografía recomendada para cada uno de estos módulos. Paralelamente, la Asociación Europea para Cuidados Paliativos en el 2004, publica el Task Force para le educación en CP en enfermería y en el 2012 se crea el marco africano para la formación en cuidados paliativos (7).

Así como la formación de profesionales enfermería ha crecido en las últimas décadas, la educación médica no se ha quedado atrás, pero se han llegado a conclusiones que la formación de los profesionales de salud: medicina, enfermería, psicología converjan en los dominios en cuidados paliativos y sea transversal a las disciplinas. Cabe resaltar que los marcos de referencia contienen los estándares mínimos homogéneos y es parte de la autonomía universitaria adaptar los contenidos a cada currículo. En este sentido, la Asociación Europea de Cuidados Palia- 
tivos (EAPC por sus siglas en inglés) ha considerado la educación y el entrenamiento de profesionales de la salud como fundamental en la promoción y expansión del cuidado paliativo en Europa (5). En 1992 el comité de educación, presidido por Derek Doyle, propuso recomendaciones específicas para el entrenamiento en Cuidado Paliativo. En el 2000, y en reconocimientos del trabajo colaborativo que caracteriza el contexto del trabajo colaborativo en Cuidado Paliativo, un grupo multi-profesional de expertos, revisó esas recomendaciones para la educación en Cuidado Paliativo.

\section{Protocolos del cuidado paliativo}

En 2004, se presentaron unas recomendaciones para la educación de cuidados paliativos en enfermería (EAPC 2004). Las recomendaciones para el desarrollo de un currículo de Medicina Paliativa es el resultado de un proyecto equivalente para el entrenamiento de médicos, tanto para el nivel básico (pregrado) como el especialista (postgrado), de acuerdo con las recomendaciones del Concejo Europeo acerca de la organización de Cuidados Paliativos 5,8. Las recomendaciones estaban basadas en currículos existentes formulados por los autores con una visión y perspectiva de toda Europa.

En el 2009, Alemania integró el cuidado paliativo al $100 \%$ de las facultades de Medicina, enfermería y psicología en el pregrado (9). En cuanto a los estudiantes de medicina, todos deben presentar el examen de cuidados paliativos para obtener la licencia de servicio. La formación diagnóstico y control de síntomas, bioética y normatividad, educación a la familia y cuidadores; comunicación asertiva, manejo adecuado de la verdad. En Canadá 17 Facultades de Medicina tiene aprobados los currículos en pregrado en CP y Australia en el 2005, publicó los estándares para el pregrado en profesionales de salud. Desde 2013, Tailandia consideró la necesidad de formación de los médicos en cuidados paliativos y el desarrollo servicios de atención para pacientes terminales debido a que el reingreso de los pacientes se evidenció que era por falta de formación en este campo (9).

Ahora bien, los médicos y las enfermeras juegan un rol fundamental en la fase terminal del paciente en términos de sintomáticos y de sufrimientos, pero existe una dimensión del ser humano que debe ser abordada conjuntamente con lo otro profesional de salud, como lo es la existencia y la concepción de la muerte, y sus sentimientos asociados como el amor, confianza, aceptación, autoestima, autonomía; y es el psicólogo (10). La intervención psicológica aborda la ruptura emocional y estados circunscritos como la ansiedad, aislamiento, tristeza incapacidad que a per se, contribuyen al sufrimiento, pero adicionalmente, brinda soporte a los demás miembros de la familia y equipo de atención sanitaria y está enfocada a la prevención y reducción del riesgo de desarrollar sensaciones de desmotivación, frustración o síndrome de Burn Out (11). La participación de psicología inicia con el diagnóstico, duelo anticipado y finaliza después de la muerte (12).

No hay evidencia importante acerca de la formación en pregrado del profesional en psicología en Colombia, se ha limitado a programas de especialidad $(12,19)$. Los marcos de referencias canadienses, australiano, irlandés y alemán apuntan al fortalecimiento de programas teniendo como base tres objetivos de intervención psicosocial y espiritual: fomento de recursos comunicativos del paciente, cuidadores y personal sanitarios, detección temprana de necesidades psicológicas específicas del enfermo y micromundo soporte al grupo multidisciplinario. En este sentido, en la formación del posgrado se han desarrollado guías y estándares de formación enfocados al cuidado psicosocial de los pacientes en estado terminal, dado que se reconoce un vacío puntual en este campo, razón por la cual la SECPAL, consideró crucial la formación de psicólogos y en el 2003 se aprobaron los estándares de formación en esta disciplina y se plantó la necesidad de incluirlo en el currículo de pregrado (12).

Si bien hay diferencias entre países en crear un currículo internacional, el Certificado Europeo en Psicología está ejecutando un proyecto piloto en 6 países de la Unión Europea y han establecido cinco áreas del conocimiento de práctica para este profesional: evaluación psicológica, counselling y psicoterapia, soporte al equipo de trabajo, educación e investigación. Adicionalmente, estos dominios se interrelaciones con otros de igual importancia en la estrategia paliativa como la normatividad, regulación profesional, bioética, diversidad cultural, autocuidado y políticas organizacionales (10).

Por otra parte, con respecto al campo de la nutrición para el paciente paliativo y su familia una de las inquietudes más importantes y conflictos más relevantes son los asociados a la nutrición en la fase terminal. Desafortunadamente, esta dimensión no está bien estudiada y aún persisten debates sobre si la misma se considera soporte 
de vida o prolongación de la muerte (13). Persiste la diferencia conceptual entre profesionales de equipo sanitario, por un lado el especialista se enfoca en el control de los síntomas o reducción tumoral, la nutricionista al balance energético y parámetros biológicos, mientras los cuidadores se preocupan culturalmente por sus creencias de "morir de hambre". Por su parte los paliativitas consideran la nutrición parenteral o enteral como fútil u obstinación terapéutica (14).

En cuanto a la terapia física en los cuidados paliativos, la literatura es mínima y la formación limitada; pero con el aumento de la expectativa de vida, paciente crónicos terminales y oncológicos se ha hecho necesario la filosofía de la terapia física aplicada al CP, aunque la mayor parte de estos profesionales están orientados hacia la rehabilitación curativa (15). Algunos autores consideran esta deformación académica secundaria a las propias posiciones paradigmáticas de la academia. El rol de fisioterapeuta en la estrategia paliativa está orientada a la adaptación física y acondicionamiento de soporte que permita disminuir el sufrimiento y sensaciones resultantes de la pérdida de la autonomía. Con respecto a la formación no hay formación en pregrado, ni en posgrado (15).

Los partes anteriores hacen referencia a los atributos de los que conforman el equipo de atención primaria al final de la vida, así como los objetivos de su participación, pero no todo sufrimiento se resuelve con fármacos o terapia. El paciente paliativo se aventura en una crisis existencial que pocos profesionales de salud están preparados para enfrentar y manejar (16). El guía espiritual recibe de los demás miembros del equipo, médicos, enfermeras, psicólogo y terapeuta impresiones e interpretaciones sobre la situación clínica del paciente. También recibe información inicial sobre su entorno familiar. Se integra dentro del equipo de cuidados paliativos entrando en diálogo con los demás miembros del equipo y trabajando de sincrónico con todos. Busca el contacto con todos, asequible y cercano, su presencia en el equipo contribuye a resaltar la importancia de la atención integral del paciente en cuidados paliativos (17). La resistencia actual a trabajar con la dimensión espiritual recae en la visión reduccionista de los profesionales del equipo sanitario, ya que este dominio no se basa en leyes, teoría o protocolos. Sin embargo, todos los individuos tienes diversas formar de explicar y comprender la existencia.
Debido a esta brecha, es que el Consorcio de Capellanes y la SECPAL, trabajan en un modelo de cuidados espirituales para los cuidados paliativos con tres preceptos fundamentales: 1) la existencia superior, 2) la conexión, 3) mediadores humanos de la experiencia espiritual (12, $16,18)$. La primera, asume la existencia la suprema sin importar la religión de paciente: Dios, Cosmos, Madre Naturaleza, Nirvana, por mencionar algunos. La segunda hace referencia a la conexión de individuo con el interior y el exterior; esta conexión se hace a solas, con el grupo familiar o por medio de rituales culturo-religiosos y está en relación con los dominios del dolor total contemplados por Saunders: físico, social, psicológico y espiritual. Y el tercero, está en relación a los humanos y el como mediadores entre el Yo interno del paciente con el exterior y permite disminuir las emociones derivadas del sufrimiento.

\section{Conclusiones}

En conclusión, se puede considerar que hay un número importante de programas en las carreras de salud que han incluido los CP a sus planes de estudio y parcialmente a los sistemas de salud; sin embargo, hay una diferencia evidente en los niveles de formación y disciplinas que participan en la estrategia paliativa, estando ligados a la normatividad de cada país. Si bien, se ha hecho un llamado a la integración transdisiciplinar de los CP en la academia y los sistemas de atención sanitaria en primer nivel, el debate aún persiste espacialmente en aspectos bioéticos como la eutanasia, limitación al esfuerzo terapéutico, obstinación terapéutica, nutrición y rehabilitación de soporte, así como la homogenización de los programas en pre y postgrado en Medicina, Enfermería y Psicología en Latinoamérica; y a pesar de la política de la OMS, aun no se han incluido los CP en los programas de pregrado en salud y el acceso a posgrado es limitado, así como la investigación.

\section{Referencias}

1. F. Mahin-Babaei, et al., 2015. The basis, ethics and provision of palliative care for dementia: A review, Maturitas.

2. Fitch1 MI, (2015) Nursing perspectives on palliative care 2015, Ann Palliat Med;4(3):150-155

3. Van Riet Paap et al. (2015). Strategies to implement evidence into practice to improve palliative care: recommendations of a nominal group approach with expert opinion leaders. BMC Palliative Care (2015) 14:47. 
4. Atlas de Cuidados Paliativos en Latinoamérica (2013). Organización Panamericana de la Salud. www.ops.org.

5. Carrasco et al., 2015. Palliative Care Medical Education in European Universities: A Descriptive Study and Numerical Scoring System Proposal for Assessing Educational Development Journal of Pain and Symptom Management 2015 (50) 516-523.

6. Baumann et al. The ethical and legal aspects of palliative sedation in severely brain-injured patients: a French perspective. Philosophy, Ethics, and Humanities in Medicine 2011, 6:4.

7. Ferrell et al. End of life nursing education (ELNEC) Ann Palliat Med (2015). 4(2):61-69.

8. Reville and Foxwell (2014). The global state of palliative care Ann Palliat Med;3(3):129-138. Ilse et al. Undergraduate education in palliative medicine in Germany: a longitudinal perspective on curricular and infrastructural development. BMC Medical Education (2015) 15:151.

9. Ortega R et al. (2005). Intervención psicológica en cuidados paliativos: revisión y perspectivas Clínica y Salud. (2005).16 (2):143-160.

10. Freeman et al. Care planning needs of palliative home care clients: Development of the interRAI palliative care assessment clinical assessment protocols (CAPs). BMC Palliative Care, (2014) 13:58

11. Fernández M. Grupo de trabajo de formación de psicólogos (SECPAL). Estándares de formación psicológica en Cuidados Paliativos. 2004,11(3); 174-179.
12. Pazart et al. Parenteral nutrition at the palliative phase of advanced cancer: the ALIM-K study protocol for a randomized controlled trial, (2014). 15:370.

13. Ganon et at. A prospective evaluation of an interdisciplinary nutrition-rehabilitation program for patients with advanced cancer Curr Oncol, (2014). 310-318.

14. Astudillo W., Mendinueta C. El síndrome del agotamiento en los Cuidados Paliativos. En: Cuidados del enfermo en fase terminal y atención a su familia., 4 Ed, 2002, 514-525.

15. Emanuel et al. Workings of the human spirit in palliative care situations: a consensus model from the Chaplaincy Research Consortium. BMC Palliative Care (2015) 14:29.

16. Balboni et al. Nurse and Physician Barriers to Spiritual Care Provision at the End of Life. Journal of Pain and Symptom Management. 2014, 48(3):400.

17. Solari et al. Home-based palliative approach for people with severe multiple sclerosis and their cares: study protocol for a randomized controlled trial. 2015. 16:184.

18. The Economist Intelligence Unit. The 2015 Quality of Death Index. Ranking Palliative care across the world. 2015; 1-71.

19. Ilse et al. Undergraduate education in palliative medicine in Germany: a longitudinal perspective on curricular and infrastructural development. BMC Medical Education (2015) 15:151. 